\title{
First on-sky results of the CAWS wavefront sensor on the CANARY experiment
}

Dubost, Nicolas, Bharmal, Nazim, Langlois, Maud, Loupias, Magali, Schotte, Clément, et al.

Nicolas Dubost, Nazim Bharmal, Maud Langlois, Magali Loupias, Clément Schotte, Michel Tallon, Lazar Staykov, Lișa Bardou, James Osborn, Fanny Chemla, Matthieu Cohen, Tristan Buey, Éric Gendron, Tim Morris, "First onsky results of the CAWS wavefront sensor on the CANARY experiment," Proc. SPIE 11448, Adaptive Optics Systems VII, 1144819 (13 December 2020); doi: 10.1117/12.2561326 


\title{
First on-sky results of the CAWS wavefront sensor on the CANARY experiment
}

\author{
Nicolas Dubosta ${ }^{\mathrm{a}, \mathrm{b}}$, Nazim Bharmal ${ }^{\mathrm{b}}$, Maud Langlois ${ }^{\mathrm{c}}$, Magali Loupias ${ }^{\mathrm{c}}$, Clément Schott $^{\mathrm{c}}$, \\ Michel Tallon ${ }^{\mathrm{c}}$, Lazar Staykov ${ }^{\mathrm{b}}$, Lisa Bardou ${ }^{\mathrm{b}}$, James Osborn ${ }^{\mathrm{b}}$, Fanny Chemla ${ }^{\mathrm{d}}$, Matthieu \\ Cohen $^{\mathrm{d}}$, Tristan Buey ${ }^{\mathrm{e}}$, Éric Gendron ${ }^{\mathrm{e}}$, and Tim Morris ${ }^{\mathrm{b}}$ \\ ${ }^{a}$ Optical Manipulation Group, School of Physics \& Astronomy, North Haugh, St Andrews, \\ KY16 9SS,UK \\ ${ }^{\mathrm{b}}$ Centre for Advanced Instrumentation, Department of Physics, Durham University, Durham, \\ DH1 3LE, UK \\ ${ }^{\mathrm{c}}$ Centre de Recherche Astrophysique de Lyon, 9 avenue Charles André, 69230 Saint Genis \\ Laval, France \\ ${ }^{\mathrm{d}}$ GEPI, Observatoire de Paris, 5 place Jules Janssen, 92195 Meudon Cedex, France \\ ${ }^{e}$ LESIA, Observatoire de Paris, 5 place Jules Janssen, 92195 Meudon Cedex, France
}

\begin{abstract}
Point-diffraction interferometers are a class of wavefront sensors which can directly measure the phase with great accuracy, regardless of defects such as vortices and disconnected apertures. Due to these properties, they have been suggested in applications such as cophasing of telescope segments, wavefront sensing impervious to the island effect and high-contrast $\mathrm{AO}$ and imaging. This paper presents an implementation of this class of interferometer, the Calibration \& Alignment WFS (CAWS), and the results of the first on-sky tests in the visible behind the SCAO loop of the CANARY AO experiment at the William Herschel Telescope. An initial analysis of AO residuals is performed in order to retrieve the SNR of interference fringes and assess the instrument's performance under various observing conditions. Finally, these results are used to test the validity of our models, which would allow for rapid implementation-specific modelling to find minimum-useful flux and other CAWS limits.
\end{abstract}

Keywords: Point-diffraction interferometry, wavefront sensing, adaptive optics.

\section{INTRODUCTION}

Future optical astronomy poses a series of technical challenges for active and adaptive optics (AO). For example, Extremely Large Telescopes (ELTs), some $10 \mathrm{~m}$ class telescopes, such as the Gran Telescopio Canarias, and even the James Webb Space Telescope have segmented primary mirrors. In order to perform as single monolithic mirror telescopes, active optics and wavefront sensing systems are required. ${ }^{1-3}$ Scientific cases enabled by future telescopes telescopes in conjunction with technologies such as eXtreme Adaptive Optics (XAO) also bring new challenges. A prominent example is the study of exo-planets. In systems such as the Gemini Planet Imager, ${ }^{4}$ it is estimated that non-common path aberrations (NCPA) between the imaging arm and the wavefront sensor (WFS) should be corrected to less than $10 \mathrm{~nm}$ rms in order to achieve direct exo-planet detection. Furthermore, XAO systems need to achieve contrast levels of between $10^{-7}$ to $10^{-9}$ around 50 mas and at rates of $2-4 \mathrm{kHz},{ }^{5,6}$ which require residual wavefront errros (WFE) to be lower than $\lambda / 280$ and $\lambda / 2800$ respectively, ${ }^{7}$ or between $6.0 \mathrm{~nm} \mathrm{rms}$ and $0.6 \mathrm{~nm}$ rms in the H-band $(\lambda=1.65 \mu \mathrm{m})$.

Point-Diffraction Interferometers (PDIs) such as the Zernike Wavefront Sensor (ZWFS) ${ }^{8}$ and the self-referenced Mach-Zehnder ${ }^{9}$ have been proposed to tackle some of these challenges. Instead of performing indirect sensing like Shack-Hartmanns (SHs), PDIs directly measure the phase without numerical reconstruction from slopes.

Further author information: (Send correspondence to N.D)

N.D.: E-mail: nsd4@st-andrews.ac.uk, Telephone: +44 (0)1334 461655

Adaptive Optics Systems VII, edited by Laura Schreiber, Dirk Schmidt, Elise Vernet, Proc. of SPIE Vol. 11448, 1144819 - (C) 2020 SPIE - CCC code: 0277-786X/20/\$21 - doi: 10.1117/12.2561326 
Thanks to this, they have been found to be robust against optical vortices and other discrete phase errors, ${ }^{10}$ and were shown to be more robust than pyramid WFSs, SHs and curvature sensors at phasing disconnected segments by ESO's Active Phasing experiment. ${ }^{11,12}$

Due to their nano-metric accuracy, PDIs have also been suggested to address the problem of accurately measuring quasi-static aberrations ${ }^{8,13,14}$ and enhancing XAO. ${ }^{9,15}$ Some PDIs have been successfully tested on telescope-based systems to characterise quasi-static aberrations, like ZELDA at the VLT-SPHERE instrument. ${ }^{16}$ This leaves XAO as one of the main challenges where PDIs are yet to produce promising laboratory and on-sky results.

An important limitation preventing PDIs from becoming the main WFS in XAO systems is their lack of dynamic range due to phase ambiguity, without which the can not overcome aberrations produced by atmospheric turbulence and close a control loop. In a previous paper we presented a novel concept called the pupil-modulated PDI (m-PDI), ${ }^{17}$ which presents an enhanced real-time (i.e. single-frame) dynamic range. With a real-time range of $\pm \pi$, instead of the $\pm \pi / 2$ range of other PDIs, the m-PDI can make use of algorithmic phase unwrapping to go beyond its natural limit. This added capability makes it a good candidate for XAO among PDIs.

The ultimate validation for any astronomical instrument comes from testing it on a telescope, with real onsky targets. This is because, despite all the rigorous lab experimentation, there will always be conditions that are either difficult to reproduce or that are just unforeseen. In an unexpected turn of events, the CANARY ${ }^{18}$ experiment at the William Herschel Telescope (WHT) received funding from the OPTICON program ${ }^{19}$ to serve as an adaptive optics (AO) testbed for new instrumentation concepts. We were awarded observation time on this new CANARY run through a joint proposal with the Centre de Recherche Astrophysique de Lyon (CRAL), in order to test the first incarnation of the m-PDI concept, the Calibration and Alignment WFS (CAWS), alongside their own novel PDI, the solid or integrated Mach-Zehnder (iMZ) interferometer. ${ }^{20}$ The iMZ is an upgraded implementation of the self-referenced Mach-Zehnder concept. Since both these instruments belong to the PDI family and share many similarities, they have comparable requirements for their respective validations. This means they can be tested at the same time by splitting the light's path, which allows us to streamline many aspects of the preparations and observations, cutting the necessary off and on-sky experiment time per instrument by about half.

The purpose of the mission is to expose the CAWS prototype to evolving distributed turbulence, to the quasistatic and closed-loop residual aberrations of an actual AO system and to the telescope operational environment. This last factor could include a variety of phenomena such as telescope vibrations, changes in gravity vectors and flexions, pupil shifts induced by the derotator, temperature variations, dome turbulence, rotating spiders, the low wind effect, atmospheric chromatic dispersion and the stars' spectral profile, amongst others. Simulating comprehensively and precisely all of these phenomena in the lab can be difficult or even impossible. For this reason, submitting the instruments to these conditions is an essential precursor to any planned deployment.

This publication is devoted to presenting the CAWS' telescope run on CANARY, which is divided into two major phases. The first phase consists of off-sky measurements of quasi-static aberrations and their compensation. This phase is a necessary prerequisite before moving onto the second phase: the measurements of post AO residual aberrations, which were performed between the nights of the $17^{\text {th }}$ and the $21^{\text {st }}$ of July, 2019. The purpose of this phase is to demonstrate how well the CAWS is able to handle the dynamic range of these aberrations, all while being fed polychromatic light, and to draw design lessons for future implementations of the instrument.

\section{WORKING PRINCIPLE}

We briefly recall the m-PDI principle ${ }^{17}$ on which the CAWS instrument relies to measure wavefront aberrations. At the entrance pupil, the aberrated wavefront is split into at least two by a small-angle beam splitter, as presented in Figure 1. In the CAWS, this element is a diffraction grating which produces an infinite number of diffraction modes. These modes are focused onto a focal plane filter mask, which only allows mode $0\left(M_{0}\right)$ and mode $+1\left(M_{+1}\right)$ through. Furthermore, $M_{0}$ is filtered by a narrow pinhole in the mask in order to produce a flat reference beam. $M_{+1}$ goes through a larger aperture which filters all spatial frequencies to prevent cross-coupling, resulting in the test beam. Both beams are collimated and then interfered at the exit pupil on Plane C. 


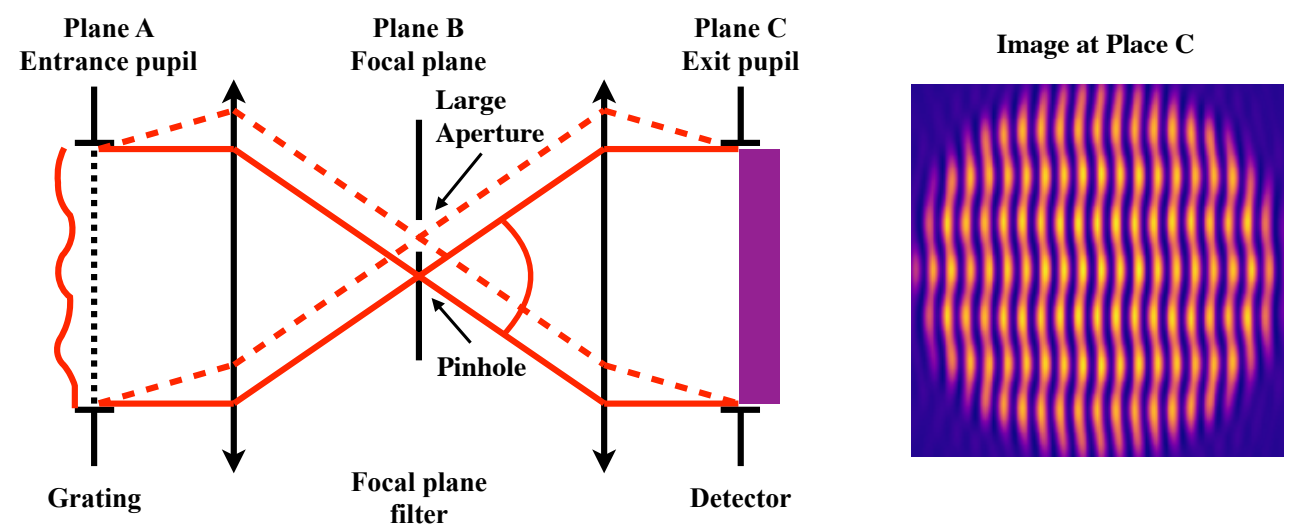

Figure 1. (left) Layout of the pupil-modulated point-diffraction interferometer. The grating splits the beam into modes. Mode 0 , shown in a solid red line, goes through a point-diffraction pinhole in the focal plane. Mode +1 goes through a larger aperture. (right) Simulated interferogram on the detector for a vertically sinusoidal wavefront.

The resulting interferogram comprises fringes modulated by the incoming wavefront. The simplified expression for the intensity of the interferogram is

$$
I_{C}(x, y)=P_{0}^{2}+\frac{1}{\pi^{2}} P^{2}+\frac{2 P_{0}}{\pi} P \cos \left(\frac{2 \pi}{T} x-\varphi_{L P}(x, y)\right)
$$

where $P$ and $\varphi_{L P}$ are respectively the amplitude and the low-pass filtered phase of the electric field $\Psi_{0}$ at the entrance pupil, $P_{0}$ is the average amplitude of the reference beam, and $T$ is the period of the interference fringes. This amplitude is in turn computed as

$$
P_{0}=P b_{0} \sqrt{S}
$$

where $S$ is the Strehl ratio on the focal plane and $b_{0}$ is a number between 0 and 1 representing the fraction of the total amplitude, determined by the geometry of the pinhole and by the small-angle beamsplitter. In order to retrieve the phase $\varphi_{L P}$, the interferogram's intensity $I_{C}$ can be demodulated by performing a Fourier transform and retrieving a sideband. Performing a further Fourier transform on it yields an estimation of the electric field, from which the phase $\varphi_{L P}$ can be computed.

\section{EXPERIMENTAL SETUP \& REQUIREMENTS}

The CANARY bench has an on-axis visible light 14-by-14 subapertures SH called the Truth Sensor (TS). In its current configuration for the OPTICON run, the TS works in conjunction with a tip-tilt mirror and a 241actuator ALPAO DM, which run the natural guide star (NGS) single-conjugate AO (SCAO) loop. As shown in Figure 2-(a), our experimental setup taps into the TS's path with a 10R/90T beamsplitter; i.e. $10 \%$ gets reflected into the TS and $90 \%$ gets transmitted into our sensors. Because of this, both the TS and our experiments are in a closed-loop configuration and can therefore receive either uncorrected wavefronts, tip-tilt corrected wavefronts (by only using the tip-tilt mirror) or fully corrected SCAO residuals. This allows us to probe the performance and the utility of these PDIs under all these situations.

Between both PDIs sits another 10R/90T beamsplitter which feeds a PSF imaging camera. This configuration allows us to perform simultaneous observations, with all 4 cameras (CAWS, iMZ, PSF imaging camera and TS) working in parallel. An image of the setup which includes all of the instruments, except for a small section of the TS, can be seen in Figure 2-(b).

Figure 3 shows the optical layout of the CAWS, as originally designed for testing on the CHOUGH AO bench. ${ }^{21}$ In this design the small-angle beamsplitter is a Ronchi diffraction grating that fits 52.7 line-pairs in the pupil of diameter $D=10.54 \mathrm{~mm}$, as provided by CHOUGH. This gives it a spatial sampling of 17.5 cycles/pupil, which corresponds approximately to the resolution of a 34-by-34 SH. The camera at the exit pupil is Imperx Bobcat B0620M with $7.4 \mu \mathrm{m}$ pixels, resulting in an oversampled 6.8 pixel/line-pair sampling. 


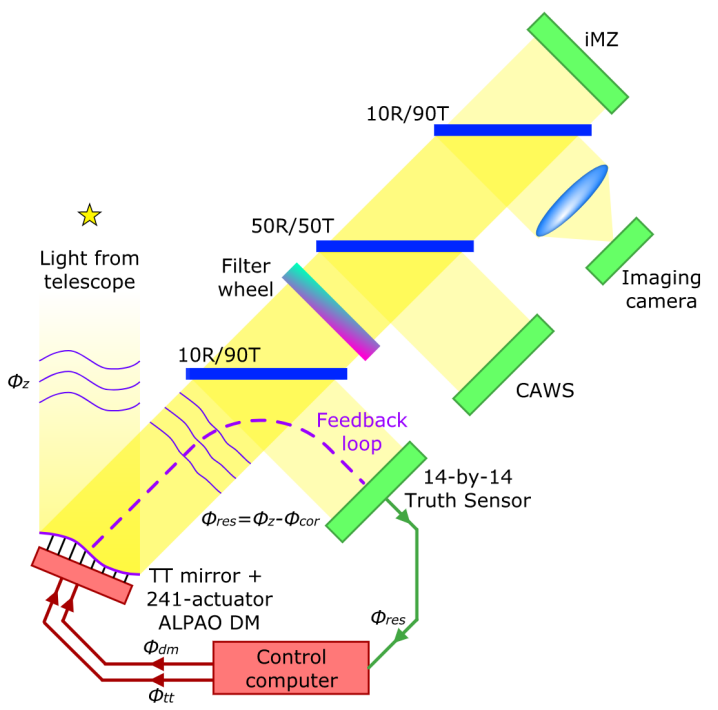

(a)

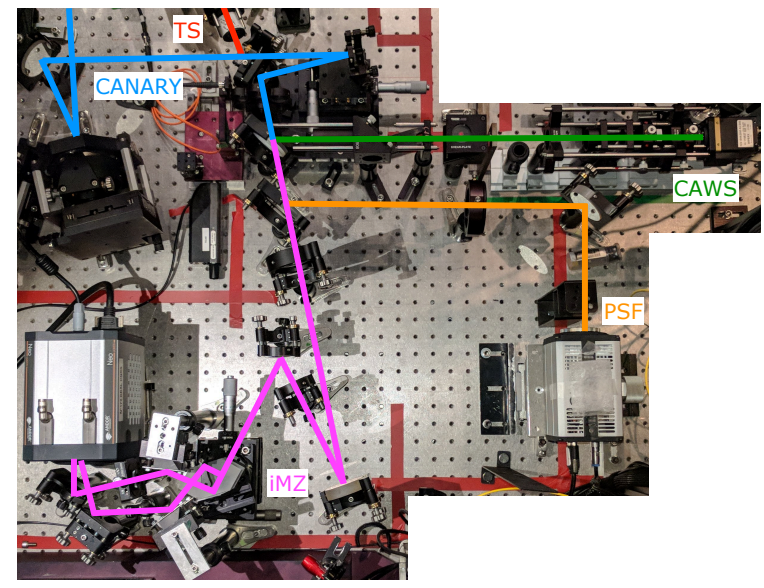

(b)

Figure 2. (a) CANARY's SCAO loop, with CAWS, an imaging camera and the iMZ, and (b) image of the experimental setup, showing only a portion of CANARY and the TS.

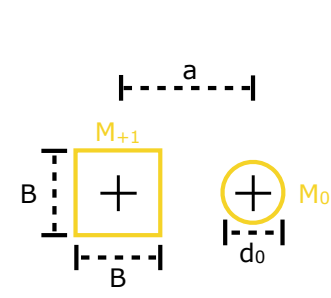

(a)

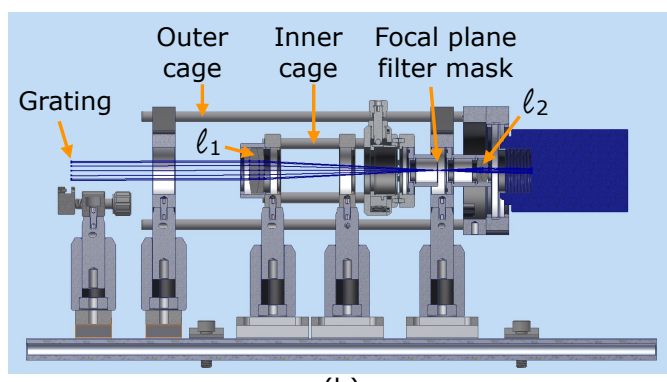

(b)

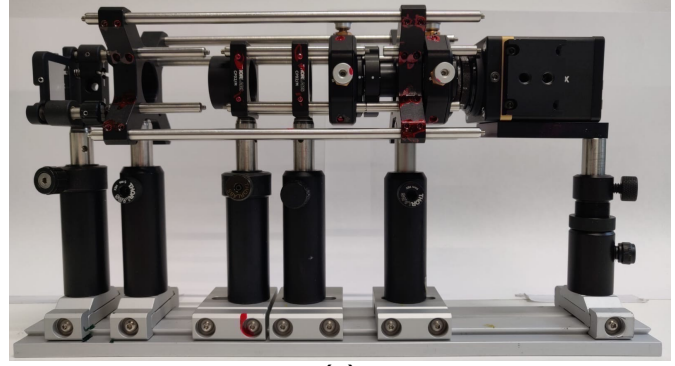

(c)

Figure 3. (a) Diagram of the pinhole and aperture on the focal plane filter mask, (b) CAWS' optical layout and mechanical assembly, and (c) image of the physical realisation.

This spatial sampling is much larger than both that of the 14-by-14 subaperture TS, and than the one needed to sample CANARY's 241-actuator DM. Having a spatial sampling larger than needed means that photons are wasted in high frequency terms that no other instrument can measure. A quick way of reducing the spatial sampling of the instrument that stays within our financial and time budgets is to resize the entrance pupil using a $4 \mathrm{~F}$ relay. Doing this also reduces the size of the exit pupil, increasing the photon count per pixel. With a resized beam diameter of $5.67 \mathrm{~mm}$, there are 28.4 line-pairs in the pupil, resulting in the equivalent resolution of approximately a 19-by-19 SH (18.9 subapertures across). These and other configuration parameters for CAWS are listed in Table 1.

With the resized pupil, the pinhole of diameter $d_{0}=16$ has a new angular size of $1.35 \lambda_{0} / D$, for a central wavelength $\lambda_{0}=675 \mathrm{~nm}$. With this pinhole size $b_{0}=0.29$. From combining Eq. 1 and 2, when $S \simeq 1$ the visibility of interference fringes is

$$
V=\frac{2 b_{0}}{\pi b_{0}^{2}+1 / \pi}=0.96
$$

The square aperture for $M_{+1}$ has a side $B=35.1 \lambda_{0} / D$. As a result, CAWS has a maximum chromatic bandwidth of $66 \%$ with respect to its central wavelength, spanning from $\lambda_{\min }=450 \mathrm{~nm}$ to $\lambda_{\max }=900 \mathrm{~nm}$.

Given the low SR delivered by CANARY in the visible, the objective that remains the most ambitious is that of measuring on-sky closed-loop residual aberrations. Since all analytical models developed until this point consider small aberrations to some degree, and by extension a relatively high SR, it is difficult to predict the 
Table 1. Configuration parameters for CAWS

\begin{tabular}{|l|c|}
\hline Name & Value \\
\hline Grid density & $5 \mathrm{lp} / \mathrm{mm}$ \\
Entrance pupil diameter & $5.67 \mathrm{~mm}$ \\
Line-pairs in pupil & $28.37 \mathrm{lp} /$ pupils \\
Equivalent SH sampling & $19 \times 19$ subapertures \\
Central wavelength $\lambda_{0}$ & $675 \mathrm{~nm}$ \\
Pinhole diameter & $16 \Leftrightarrow 1.35 \lambda_{0} / D$ \\
Maximum fringe visibility & $0.96 @ \lambda_{0}$ \\
Fringe sampling & 6.9 pixels $/ \mathrm{lp}$ \\
Pixels in pupil & 30,095 pixels \\
\hline
\end{tabular}

behaviour of the CAWS when faced with strong aberrations and low SR. The opportunity to go on-sky and test the CAWS in extreme conditions, exploring the circumstances under which our theoretical models break down and how to extend them to make them more robust. By doing so, new insights could be drawn that could help us more accurately determine the minimum requirements on any future hosting AO system, and in turn to find designs that better suit them.

\section{PHASE 0: CHARACTERISATION OF QUASI-STATIC ABERRATIONS}

Before the PDIs can be tested on-sky, the aberrations on the wavefronts reaching them need to be minimised and the reference slopes set on the TS. This will offload most of the non-common path aberrations (NCPAs) onto the TS which has a greater dynamic range, in turn enhancing the dynamic range of the PDIs. Since the imaging camera sits between the CAWS and the iMZ, by symmetry, minimising the static aberrations on this camera does not grant an advantage to any one PDI over the other. Therefore, it was agreed that quasi-static aberrations would be calibrated for by sharpening the PSF on that camera, and then recording references on all other WFSs.

After the PSF was sharpened, reference slopes were recorded on the TS and then used to close the control loop on an internal calibration source. This ensures that the DM keeps a stable shape, while the PDIs can record reference phases of their own. The reference phases on CAWS are as shown on Figure 4. A $635 \mathrm{~nm}$ diode laser
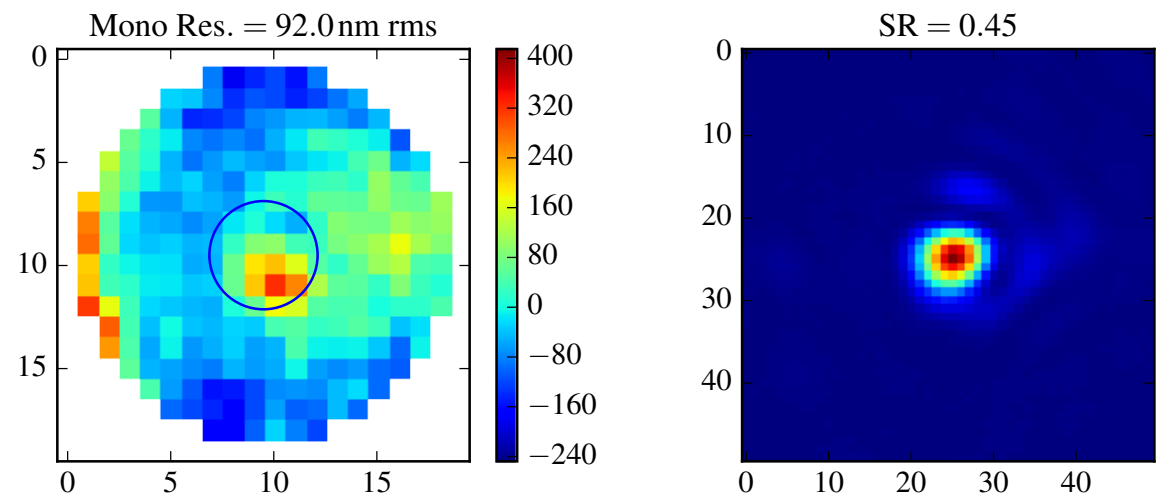

Figure 4. (left) Residual static aberrations on CAWS in nanometers and (right) corresponding PSF image measured on its focal plane mask, mode +1 . Reference phases and focal plane measurements where produced using the $635 \mathrm{~nm}$ diode laser internal source.

source was used to record phases because its narrowband spectrum facilitates the productions of fringes in case the aberrations were still too large to be measured with broadband polychromatic light. Furthermore, all the optics were aligned with this source, as it provides great flux and enough coherence to collimate beams using 
a shear plate. As a result, both PDIs should see the least amount of defocus at this wavelength, while other wavelengths should suffer from some chromatic aberrations. After acquiring references, an additional positive lens was added to CAWS in order to image a PSF on its focal plane. Since this PSF goes through the aperture on the mask corresponding to the diffraction mode +1 , it is only being filtered at very high spatial frequencies, where almost no light remains after the initial sharpening, which has concentrated most light around the PSF's core and first few diffraction rings. As a result, it is almost identical to a PSF not being filtered around the edges. The SR measured on this image can therefore be used as a preliminary test to check that the instrument is receiving a good PSF and that most of the aberrations' power is being sensed by the CAWS in its WFS configuration. Indeed, when the standard deviation of the wavefront is converted into a SR using Maréchal's approximation, ${ }^{22}$ the resulting value is 0.436 , which only has a near $3 \%$ discrepancy with the SR calculated from the image, showing a good initial agreement.

Besides providing initial sanity checks and helping to make sure that residual static aberrations are small enough for the CAWS to be well within its dynamic range, the main purpose of these reference phases will be to distinguish atmospheric aberrations from static ones in on-sky measurements. These measurements will mostly be acquired using broadband filters, in order to maximise the amount of light reaching the instrument's detector. As shown in a previous paper, it is theoretically possible to produce accurate polychromatic phase measurements, given a known central wavelength and a relatively flat chromatic spectrum. ${ }^{17}$ But until now, there has been no experimental verification showing that the CAWS can actually do this.

A first validation that accurate broadband measurements are possible is to find a good agreement between the monochromatic and the broadband polychromatic characterisations of the same quasi-static aberrations. For this, the system is illuminated using a halogen lamp, delivered by a single mode fibre, and by using a $625 \mathrm{~nm}$ long-pass dichroic on the filter wheel (See Fig.2). This filter is used as it allows through a broadband spectrum, which is also sufficiently confined for wavefront sensing to still be possible. Figure 5 shows the interference fringes obtained with both sources. In principle, both sets of fringes, monochromatic and broadband, should have the
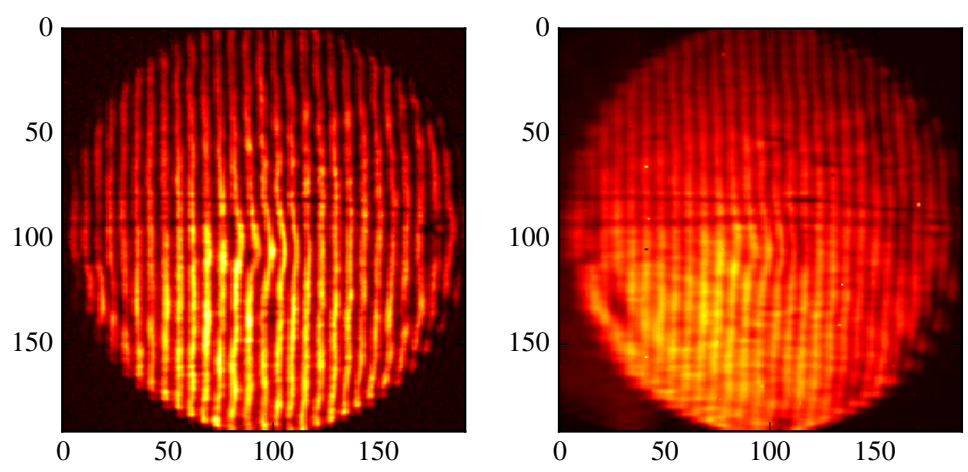

Figure 5. Interference fringes obtained using (left) a monochromatic diode laser and (right) a broadband halogen lamp and a $625 \mathrm{~nm}$ long-pass dichroic. The two horizontal and almost parallel lines across the pupil are hairs located on a conjugate plane for focusing.

same visibility. The drop in fringe visibility when using the halogen lamp is due to chromatic aberrations.

In a perfect system, the phase measurements retrieved from the broadband fringes in radians can directly be scaled into units of length, e.g. nanometers, by using an equivalent central wavelength. Normally, the only other step in this process is to estimate said wavelength, by using the CAWS in its spectrometer configuration and measuring the light source's chromatic spectrum. This configuration is achieved by adding a positive lens near the exit pupil so that the focal plane, where the diffraction grating produces chromatic dispersion on mode +1 , can be imaged. Focal plane images with both the halogen lamp and the diode laser, are presented in Figure 6. As can be seen in the top panel of the figure, light from diffraction mode +1 has a larger vertical spread than seen in Figure ??, due to compound chromatic aberrations present in the optical relay preceding the CAWS and in the CAWS itself. As a consequence, some extra steps are required before the central wavelength can be estimated from these images. 

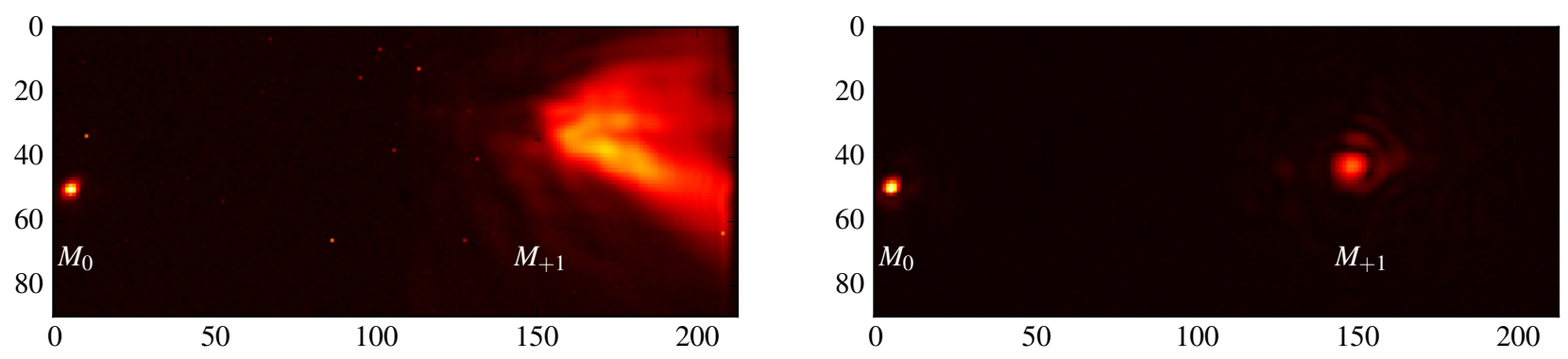

Figure 6. Focal plane images of diffraction modes 0 and +1 with (left) halogen lamp and (right) diode laser for reference. Original values have been square-rooted to increase the contrast of the images presented in this figure. The intensity in each image is normalised to its own maximum value.

As shown in Figure 7, there is a strong chromatic defocus between the wavelength of the diode laser used to focus the optics $(635 \mathrm{~nm})$ and other wavelengths spanning the range of the CAWS. On the redder edge around

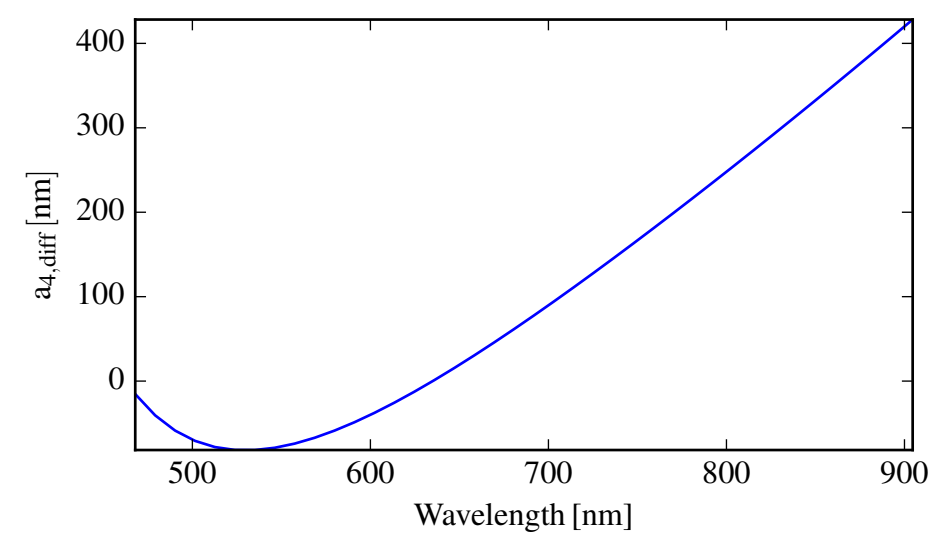

Figure 7. Zemax estimation of the differential chromatic defocus term in the CAWS' focal plane, between wavelengths spanning its chromatic range and the diode laser's wavelength $(635 \mathrm{~nm})$. The model includes CANARY and the optical interfaces leading to the CAWS.

$900 \mathrm{~nm}$, the differential defocus term can reach values around $400 \mathrm{~nm} \mathrm{rms}$. Due to this, it should be expected that longer wavelengths transmit less light through the pinhole of the focal plane mask as they become more defocused. The transmitted light should therefore have a different chromatic spectrum from the one measured on mode +1 , as it is weighted by a decreasing fraction of the total light. Since there is no chromatic dispersion on mode 0 (the mode reaching the pinhole), this spectrum can not be directly measured using the CAWS in its spectrometer configuration and, instead, needs to be reconstructed numerically.

In order to reconstruct the spectrum of mode 0 , a simulation is performed that combines three elements. The first element is the monochromatic reference wavefront already presented in Figure 4, which is assumed to be the same for all wavelengths. The second element is the chromatic defocus obtained from the optical model. These two aberrations are added into the phase of an electric field which is propagated to the focal plane, producing a PSF. For every wavelength, the resulting PSF is propagated through the pinhole, allowing us to determine the fraction of total light intensity going through it. Finally, the third element, the spectrum measured by CAWS on mode +1 , is weighted by the fractional intensities, hence reconstructing the spectrum of mode 0 . Both spectrums, as measured on mode +1 and reconstructed for mode 0, are shown in Figure 8.

Also shown in the figure is amplitude of the interference fringes as a function of wavelength, normalised with respect to it's maximum value, which is calculated as

$$
A_{\text {fringe }}(\lambda)=\left(\sqrt{I_{0}(\lambda)}+\sqrt{I_{+1}(\lambda)}\right)^{2}-\left(\sqrt{I_{0}(\lambda)}-\sqrt{I_{+1}(\lambda)}\right)^{2},
$$




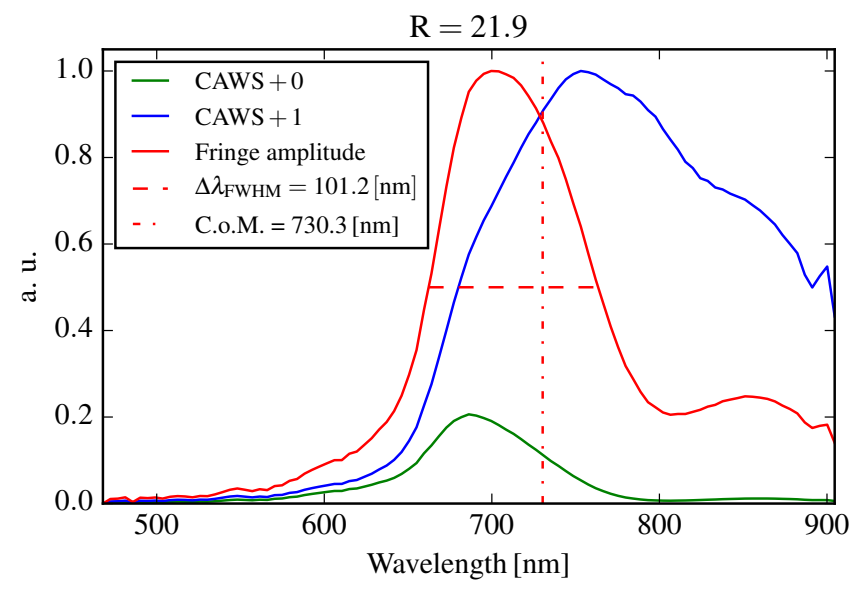

Figure 8. Chromatic spectra (blue) as measured on mode +1 and (green) as reconstructed for mode 0 . The values of these curves have been normalised to the same reference so that the maximum common value between them is equal to 1 . This normalisation preserves their relative magnitudes. Both spectrums are used to estimate (red) the normalised amplitude of interference fringes as a function of wavelength. The CoM and FWHM are presented for the fringes' amplitudes. $R$ is the theoretical spectral resolution.

where $I_{0}(\lambda)$ and $I_{+0}(\lambda)$ are the intensity of light going through apertures $M_{0}$ (pinhole) and $M_{+1}$ respectively. In the first place, this curve of amplitudes confirms that the fringes are indeed produced by broadband light, with a FWHM of $101.2 \mathrm{~nm}$, or about a $14 \%$ bandwidth with respect to the Centre of Mass (CoM). Secondly and most importantly, by calculating the CoM, an estimate for the equivalent central wavelength is derived. As the relative contribution of the fringes increases with their amplitude, it will be presumed that this value, located at $730.3 \mathrm{~nm}$, is a useful representation of our target parameter.

After scaling the measurements with the estimated central wavelength, it still remains to subtract the differential defocus term. As mentioned earlier, chromatic aberrations add a differential defocus between separate wavelengths that need to be accounted for. The broadband references, corrected for differential defocus, are presented on Figure 9, side by side with the monochromatic references for comparison, from Figure 4, and the phase error between them. The error between the monochromatic and the broadband references is $23.6 \mathrm{~nm} \mathrm{rms,}$
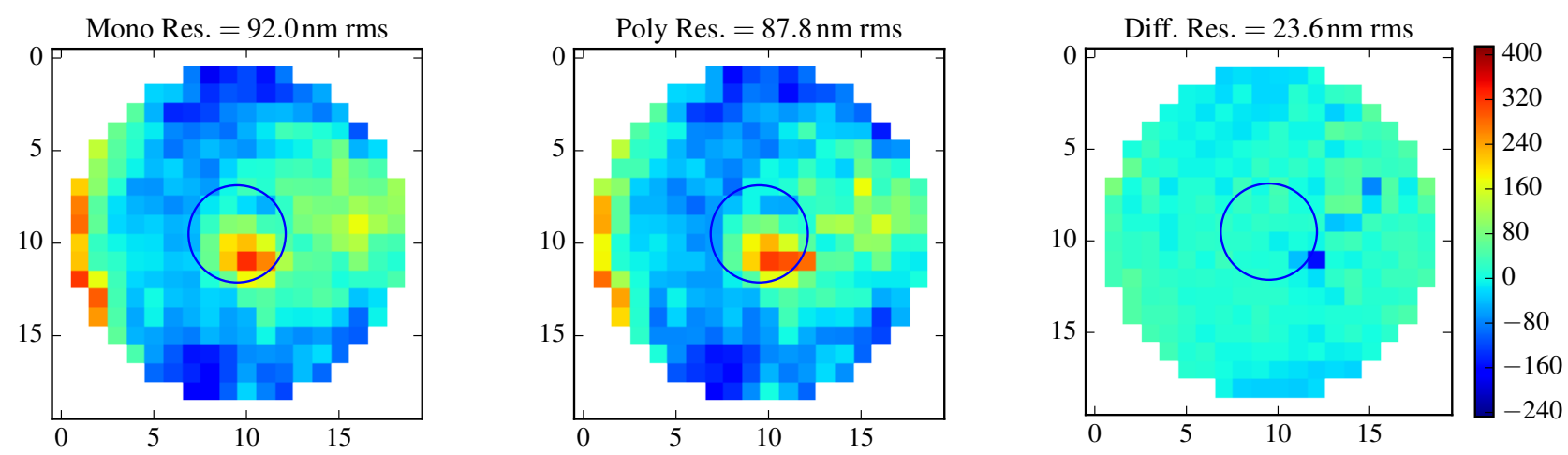

Figure 9. (left) Monochromatic references, (centre) broadband references, corrected for differential defocus, and (right) error between them, all in nanometers. Blue central circles mark the location of the telescope's central obscuration.

or about $26 \%$ of the total static aberrations measured with the diode laser. On the one hand, this is not a negligible amount and more study is needed before this error can be systematically accounted for in the instrument's error budget. Further tests could also show if at least part of this error arises from changing light sources on the SCAO loop, leading to a different performance of the loop. On the other hand, these initial results show that the general shape of the static aberrations can be correctly determined with broadband light by simply scaling 
the phase measurements with the appropriate wavelength and then correcting for the chromatic aberrations.

Being able to do so is a pre-requisite for the correct scaling and defocus correction of on-sky measurements, such as those presented in the following section.

\section{SNR OF INTERFERENCE FRINGES}

After successfully measuring and calibrating for static aberrations, the experiment is ready to enter Phase 1 and acquire on-sky data. But producing fringes that have a sufficiently high SNR to be detectable will be challenging even in the best conditions, given CANARY's low SR in the visible of approximately $0.5 \%$ at $675 \mathrm{~nm} .{ }^{23}$ As a consequence, the main focus of these observations will be to establish the operational parameters that maximise the appearance of fringes. This will be achieved by controlling and sweeping over two parameters, the chromatic bandwidth and the exposure time, all while keeping the main SCAO loop closed on a bright star. In the case of the chromatic bandwidth, the results will show the optimal solution when broadening the spectrum in the trade-off between allowing more light through to the detector at the cost of more chromatic defocus and poorer visibility. With respect to exposure time, the trade-off is between the amount of light collected and the loss of visibility due to the averaging out of fringes. But in order to find these optimal values, a metric that is representative of the quality of the interference fringes needs to be defined.

A potential way to determine the quality of the interference fringes is by measuring their amplitude, relative to that of the background noise. This term, which will be considered to be the detection SNR of the fringes, is noted $\mathrm{SNR}_{f}$ and can be efficiently calculated in the Fourier transform of the exit pupil interferogram $I_{C}$, noted $\widehat{I}_{C}$. By looking inside the sideband region, defined by the mask aperture $M_{+1}$, we should find the signal of the interference fringes

$$
\begin{aligned}
\widehat{a}(k) & =\widehat{I}_{C} M_{+1} \\
& \simeq \frac{b}{\pi} \widehat{\Psi}_{L P}\left(k-\frac{\lambda_{0} f}{T}\right),
\end{aligned}
$$

where $b$ is the amplitude of light making it through the pinhole, $\widehat{\Psi}_{L P}$ is the Fourier transform of the low-pass filtered electric field, $T$ is the period of the fringes and $f$ is the focal length of the lens preceding the focal plane mask. Using Parseval's theorem, the energy of the fringes can be calculated by integrating $\|\widehat{a}(k)\|^{2}$ inside the sideband. In a noiseless scenario, this would be

$$
A^{2}=\iint_{M_{+1}}\|a(k)\|^{2} d k .
$$

Unfortunately, since the photon-shot noise and the read-out noise should be homogeneously distributed across the frequency space, $a(k)$ is inevitably found added to the Fourier transform of the sum of both noises, $\widehat{n}(k)$. The resulting integral is of the form

$$
\iint_{M_{+1}}\|a(k)+\widehat{n}(k)\|^{2} d k,
$$

where the noise term in inexorably entangled to the fringe signal term in the computation of the absolute value.

This entanglement can be partially mitigated by estimating the noise term elsewhere in the Fourier plane and then subtracting it to the final integral. This is possible because, as mentioned earlier, the noise is homogeneously distributed, which means that integrating its power in two different regions of similar surface should, on average, yield the same value. The only condition is that the region where the noise is estimated does not have any signal coming from the fringes. The power of the noise in the sideband is

$$
\delta N_{+1}^{2}=\left\langle\iint_{M_{+1}}\|\widehat{n}(k)\|^{2} d k\right\rangle,
$$

and the estimated power in a region $M_{\text {estim. }}$ with no signal from the fringes and of equal surface is

$$
\delta N_{\text {estim. }}^{2}=\left\langle\iint_{M_{\text {estim. }}}\|\widehat{n}(k)\|^{2} d k\right\rangle .
$$


Values $\delta N_{+1}^{2}$ and $\delta N_{\text {estim. }}^{2}$ are in turn random variables that are, in principle, independent and have equal average and equal variance. Consequently, the compound random variable $\delta N_{\text {diff. }}^{2}=\delta N_{+1}^{2}-\delta N_{\text {estim. }}^{2}$ has an expectation of zero and a variance $\operatorname{Var}\left(\delta N_{\text {diff. }}^{2}\right)=2 \operatorname{Var}\left(\delta N_{+1}^{2}\right)$. This new variable adds uncertainty to our further estimation of SNR. As a side note, in the case $M_{\text {estim. }}$ does not have the same surface as the sideband $M_{+1}$, the estimated noise power should be rescaled by using the ratio between both regions' surfaces, as will generally be the case with real data.

Having estimated the power of the noise, it can be subtracted to the integral of the sideband to produce a good approximation of the total fringe intensity

$$
\begin{aligned}
A_{\text {estim. }}^{2} & =\iint_{M_{+1}}\|a(k)+\widehat{n}(k)\|^{2} d k-\delta N_{\text {estim. }}^{2} \\
& =\iint_{M_{+1}}\|a(k)\|^{2}+\|\widehat{n}(k)\|^{2}+2 \operatorname{Re}\left\{a(k) \widehat{n}^{*}(k)\right\} d k-\delta N_{\text {estim. }}^{2} \\
& =\iint_{M_{+1}}\|a(k)\|^{2}+2 \operatorname{Re}\left\{a(k) \widehat{n}^{*}(k)\right\} d k+\delta N_{\text {diff. }}^{2} .
\end{aligned}
$$

In the previous expression, the conjugate value of the noise, $\widehat{n}^{*}(k)$, can be considered to have a random angle, completely uncorrelated to that of the fringes' signal $a(k)$. As a consequence, $2 \operatorname{Re}\left\{a(k) \widehat{n}^{*}(k)\right\}$ has a random sign, leading its integral to have a null average value. This, combined with the fact that $\iint_{M_{+1}}\|a(k)\|^{2} d k$ grows with the square of the signal $a(k)$, whereas the standard deviation of $\iint_{M_{+1}} 2 \operatorname{Re}\left\{a(k) \widehat{n}^{*}(k)\right\} d k$ can only grow

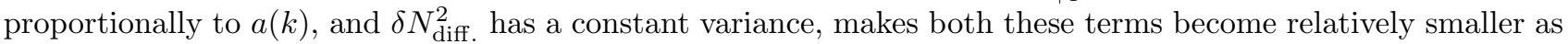
a part of $A_{\text {estim. }}^{2}$ for larger signals. This means there is a point where $A_{\text {estim. }}^{2}$ can be approximated to

$$
A_{\text {estim. }}^{2} \simeq \iint_{M_{+1}}\|a(k)\|^{2} d k=A^{2}
$$

for a large enough signals.

Now that the intensity of the fringes can be estimated, it can be used to compute the empirical SNR of the fringes

$$
\mathrm{SNR}_{f}^{(\text {empiric })}=\sqrt{A_{\text {estim. }}^{2} / \delta N_{\text {estim. }}^{2}},
$$

which is an estimation of the true SNR value

$$
\mathrm{SNR}_{f}^{(\text {true })}=\sqrt{A^{2} / \delta N_{+1}^{2}}
$$

\section{SCAO REPLAY}

The method for empirically computing the SNR of fringes is first tested by using the internal diode laser source and simulating SCAO residuals on the bench. In order to do this, residual slopes were recorded on a previous on-sky SCAO run. After subtracting the tip-tilt term, these aberrations are multiplied by the system's control matrix to produce DM commands. By passing these commands onto the DM, the residual aberrations can be emulated and replayed as they would have been seen at the backend of the AO system, albeit filtered by the DM's transfer function and had the tip-tilt term been perfectly corrected for. In this controlled environment, aberrations have less power due to the subtraction of tip-tilt and the constraints on integration time and flux are relaxed, which allows us to acquire high SNR measurements.

An example of fringes obtained with this replay is presented in Figure 10, alongside the regions in the Fourier plane being used to estimate the empirical SNR of the fringes. These images were obtained using a $2 \times 2$ binning on the detector's camera. As can be seen on the figure, the (top-left) image with the sharpest and brightest fringes, also has a brighter sideband (blue region), which in turn results in a higher SNR. These results serve as a visual confirmation that the estimation of the SNR defined above is a good representation of the overall quality of the fringes. As an added remark, the control matrix that was used to create this replay was built to 

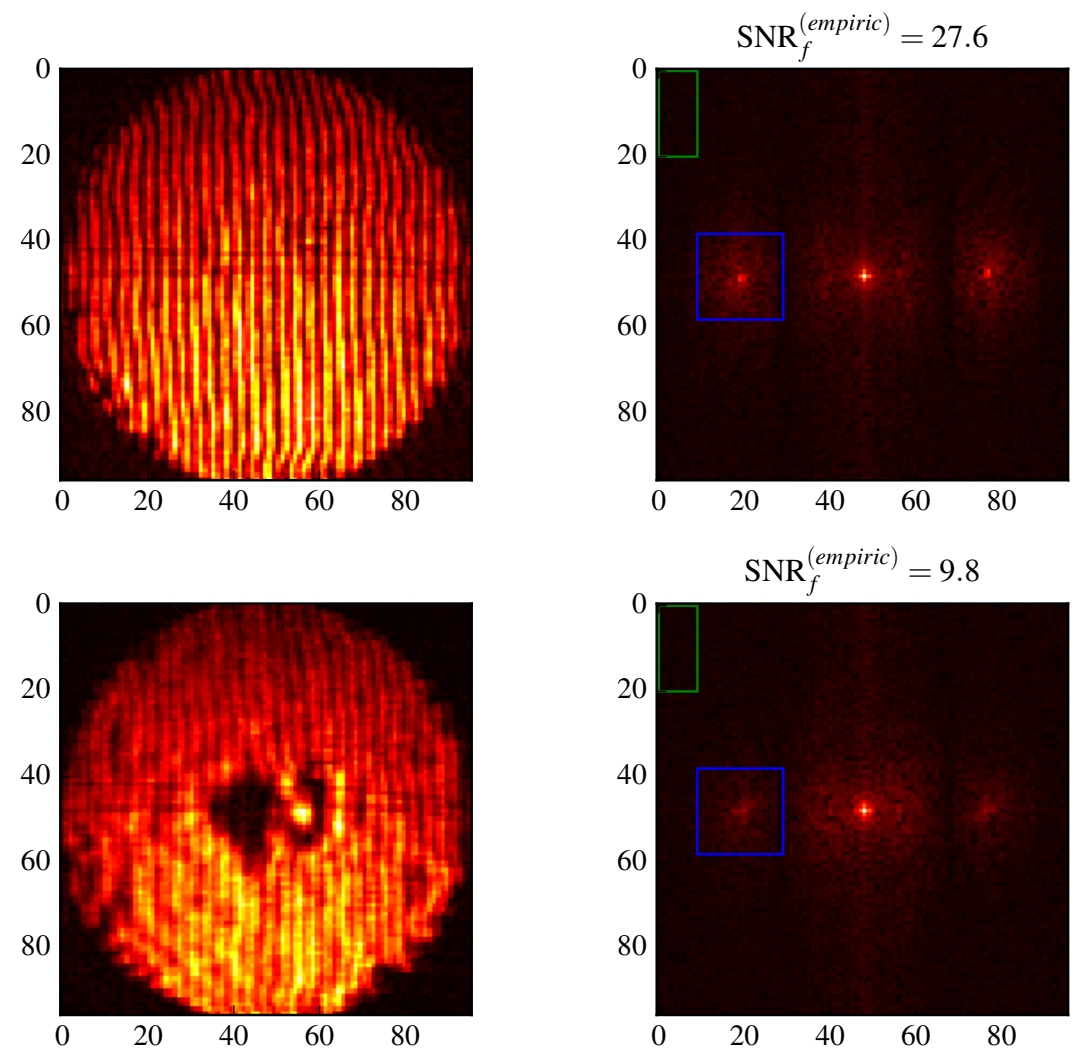

Figure 10. Off-sky (left) interference fringes with (right) their respective Fourier transform (the square-root ob the absolute value is displayed for contrast enhancing purposes). Fourier transform panels also show (blue) the sideband region $M_{+1}$, (green) the noise estimating region $M_{\text {estim. }}$, and the resulting estimation of SNR ${ }_{f}^{(\text {empiric) }}$.

be applied on-sky, where the secondary mirror of the telescope produces a central obscuration. Consequently, the control matrix can not control the actuators behind the obscuration, leading to them sometimes wandering aimlessly and introducing the great phase aberrations that are seen in the centre of the bottom-left panel.

In order to further demonstrate the relationship between the empiric SNR of the fringes and their quality, six images with widely different SNRs are selected from the replay sequence and presented in Figure 11. As can be seen in the figure, images with high SNR have sharp and well defined fringes. This is the case for the images at the top. In the opposite case, (bottom-right) images approaching an SNR around 1, where the fringes and the noise have about the same power, present at best blurry fringes in some regions, such as the bottom of the pupil, at worst regions without fringes, such as the top-left of the pupil, and sometimes regions with ambiguous fringes, such as the bottom left. Finally, notice that there are no inconsistent cases, where a higher SNR leads to clearly worst fringes, or vice-versa. This is not just true in the examples presented here, but also in all images that have been visually inspected.

\section{ON-SKY AO RESIDUALS}

With this tool in hand, it is now possible to acquire and analyse on-sky data. After several nights trying different stellar targets, exposure times and filters, only filters $1(697 \times 75 \mathrm{~nm})$ and $6(>625 \mathrm{~nm}$ dichroic) allowed for sufficient light to reach the CAWS and produce visible results on which any meaningful analysis is possible. The best results, yielding the most light and the highest SNRs were obtained during the last night with the star Beta Pegasi of magnitude 0.92 in the R band. Unfortunately, the SNR remains low, just above 1, even in the best images. Figure 12 shows a pair of the best on-sky images and their respective SNRs. Just as with the replay data, these images where obtained using $2 \times 2$ binning. As can be seen on the figure, for SNRs close to unity, 

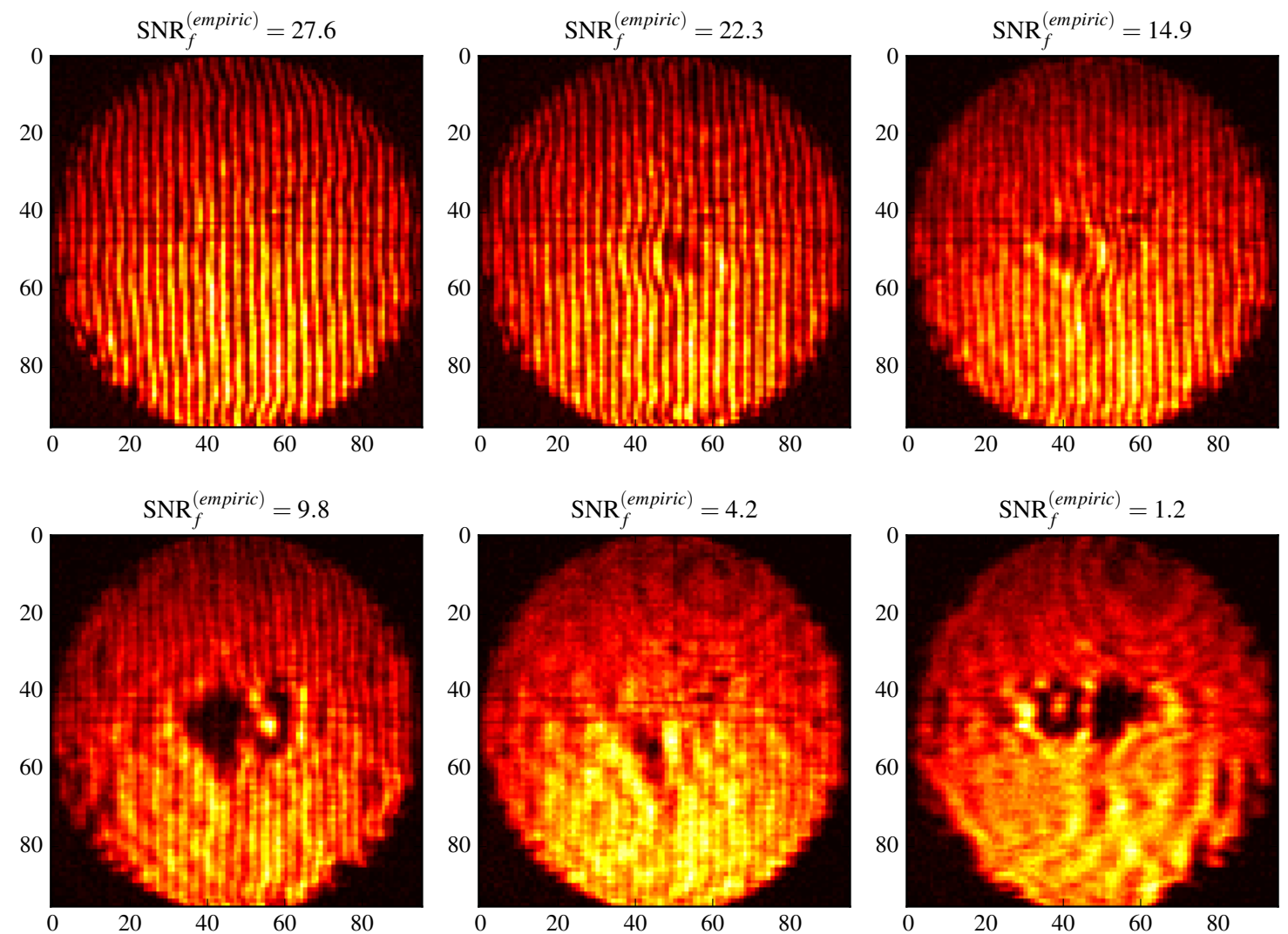

Figure 11. Off-sky sequence of images from replay data, with decreasing SNRs, from left to right and then from top to bottom.
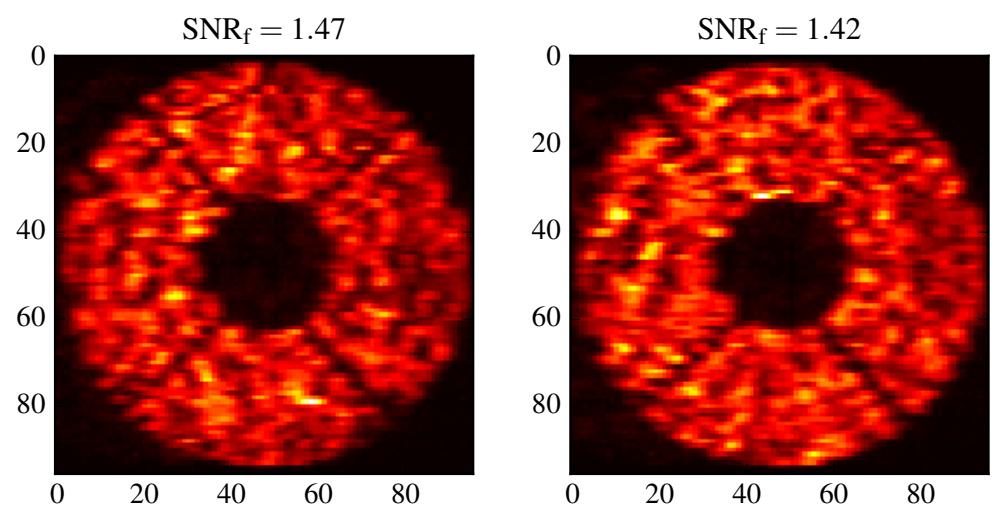

Figure 12. On-sky images with the highest $\mathrm{SNR}_{f}^{\text {(empiric) }}$. Images were produced while observing Beta Pegasi, with a ¿625 nm dichroic and an integration time of $400 \mathrm{~ms}$.

fringes remain ambiguous on raw images, i.e. it is difficult to distinguish true fringes from what could just be the result of noise. The reason for this is that at these levels, interference fringes have a power which is comparable to that of the noise.

Most of the images acquired have in fact an empirical SNR below 1. Figure 13 shows the average SNR of fringes for multiple exposure times and for two filters, as well as the expected SNR predicted by an unfitted analytical model, which is still a work in progress. From the figure, it is possible to note that SNR values obtained with filter 2 , the broader of the two, are slightly higher. This is in line with the model's prediction that an increase in overall flux $I_{0}$ also leads to an increase in SNR. 


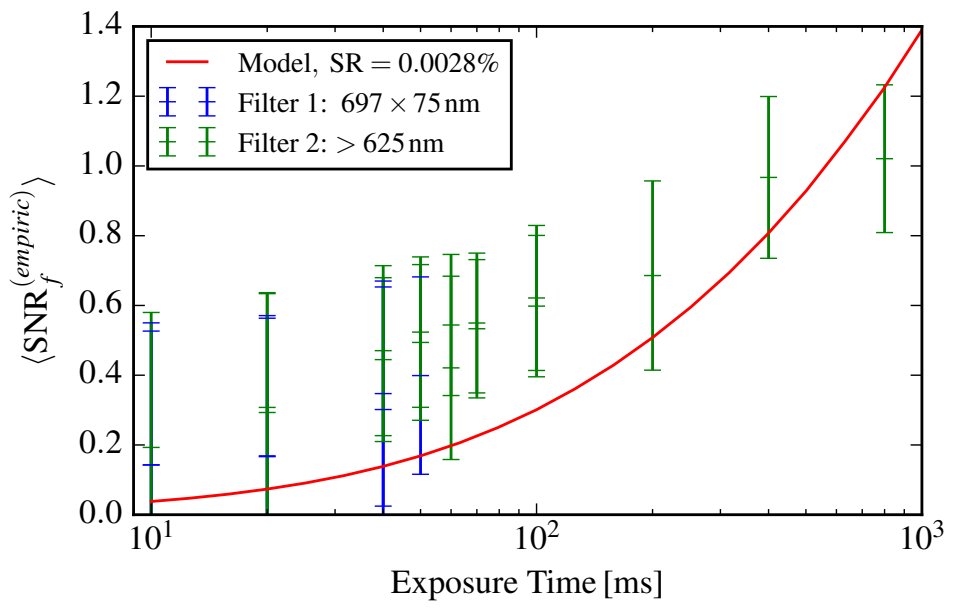

Figure 13. SNR of fringes for different exposure times and filters, and the expected value of an unfitted model for $\mathrm{SR}=0.0028 \%$. The bars extend $\pm 1.5 \sigma$ from the average value, spanning a total of $3 \sigma$. Results were produced on-sky while observing Beta Pegasi.

The SR parameter of the model was not fitted to the data, but was instead only adjusted manually to approximate it. The resulting average SR is in the vicinity of $0.0028 \%$, which for a central wavelength of $730 \mathrm{~nm}$ translates to an average RMS aberration of $376 \mathrm{~nm}$. Given that CANARY in SCAO mode can produce SRs between 0.1 and about 0.45 in the $\mathrm{H}$ band, ${ }^{23}$ which translate to between $228 \mathrm{~nm}$ and $386 \mathrm{~nm}$ RMS, then the variables and the output of the model have the right orders of magnitude. Regardless of this first sign of validity, the model fails to describe the rate at which the SNR increases with integration time. As can be seen in the figure, the average SNR only improves by about $5 \%$ between $400 \mathrm{~ms}$ and $800 \mathrm{~ms}$, despite a doubling of the integration time. In contrast, between these same intervals the model predicts an increase of more than 50\%: 10 times more than the data shows.

This poor fit is due to two reasons. The first one is that at very low SR the instantaneous PSF no longer has a single maximum, but is instead broken down into several speckles. The result is the intensity of the reference beam depends on whether one of these speckles will reach or miss the pinhole, making the concept of SR no longer meaningful at short exposure times. Since the model assumes the SR can be used to compute the intensity of the reference beam, then its validity drops hand in hand with that of this basic assumption.

The second problematic assumption is the model only considers short integration times relative to the coherence time $\tau_{0},{ }^{24}$ which according to Fusco, ${ }^{25}$ is between $1 \mathrm{~ms}$ and $20 \mathrm{~ms}$, for a wavelength of $500 \mathrm{~nm}$. For $730 \mathrm{~nm}$, $\tau_{0}$ is between $1.5 \mathrm{~ms}$ and $30 \mathrm{~ms}$. In other words, time lag has not yet been included into the error budget. When exposure times grow past this delay, as is the case for those on the right half of the figure, integrated interference fringes become the average of different wavefronts. This brings a decrease in visibility, acting against the increase in SNR and causing the slower rate of growth observed in the data.

\section{DISCUSSION}

Currently, solving the inaccuracies produced by low SRs, as well as modelling long integration times both remain unsolved problems. Producing accurate predictions of the SNR of fringes, given different atmospheric conditions, guide star magnitudes and AO specifications, is of great importance to understanding the usability and benefits of the CAWS within AO. The next step in this direction is to perform comprehensive end-to-end Montecarlo simulations of the CAWS operating in closed-loop in order to determine the best design parameters and their performance. For example, according to our still perfectible model, the low SNR reached during on-sky testing was most likely due to light not going through the pinhole. This could be fixed in several ways. The first way is to move to longer wavelengths, hence ensuring better SRs at the back of the AO and with it a better coupling of the pinhole. The second way to improve the coupling is to make the pinhole larger. During the run, the pinhole diameter was $1.35 \lambda / D$. By almost doubling this diameter to about $2.5 \lambda / D$, the amplitude of the reference beam, 
and by extension the SNR, also doubles in these low SR conditions, while still keeping good sensitivity to low order aberrations.

Simulations could also be performed to test different AO configurations. For instance, with AO systems that include a parallel low-order WFS, even larger pinholes could be tried, as there is no need for the PDI to sense low-order aberrations. PDIs could indeed find their best utility in such configurations, with two or more hierarchically arranged WFSs, each of which measures aberrations of a particular order. In this setup, the CAWS could be used as a high-order WFS, where the size of its pinhole is determined by the spatial resolution of its low-order counterparts.

If adjusting design parameters and $\mathrm{AO}$ configurations is not enough, many other upgrades can be pursued to improve the performance of the CAWS in general. For example, the current throughput of the diffraction grating is only $50 \%$. This could almost be doubled by switching to gratings with a near $100 \%$ reflection or transmission, such as reflective échelles, Volume Phase Holographic Gratings (VPHGs), or patterned liquid crystals. All of these alternatives have chromatic effects that still need to be assessed, but bring with them many advantages. In addition to having better throughput, all of these elements could be put in partially blazed configuration, removing light away from diffraction mode -1 and redirecting it towards modes 0 and +1 . This in turn, increases the throughput of the focal plane filter mask, which is currently about $40 \%$, depending on the pinhole size. Until the overall low throughput of the CAWS is improved, its relatively large chromatic bandwidth does not grant it an immediate advantage over other PDIs, regarding total flux and resulting SNR.

\section{ACKNOWLEDGMENTS}

Comisión Nacional de Investigación Científica y Tecnológica (CONICYT) (72160371); UK Research and Innovation Science and Technology Facilities Council (STFC) (ST/L002213/1, ST/L00075X/1,ST/P000541/1,ST/T000244/1).

\section{REFERENCES}

[1] Yaitskova, N., Dohlen, K., and Dierickx, P., "Analytical study of diffraction effects in extremely large segmented telescopes," JOSA A 20(8), 1563-1575 (2003).

[2] Pizarro, C., Arasa, J., Laguarta, F., Tomàs, N., and Pinto, A., "Design of an interferometric system for the measurement of phasing errors in segmented mirrors," Applied optics 41(22), 4562-4570 (2002).

[3] Acton, D. S., Knight, J. S., Contos, A., Grimaldi, S., Terry, J., Lightsey, P., Barto, A., League, B., Dean, B., Smith, J. S., et al., "Wavefront sensing and controls for the james webb space telescope," in [Space Telescopes and Instrumentation 2012: Optical, Infrared, and Millimeter Wave], 8442, 84422H, International Society for Optics and Photonics (2012).

[4] Fusco, T., Rousset, G., Sauvage, J.-F., Petit, C., Beuzit, J.-L., Dohlen, K., Mouillet, D., Charton, J., Nicolle, M., Kasper, M., et al., "High-order adaptive optics requirements for direct detection of extrasolar planets: Application to the sphere instrument," Optics Express 14(17), 7515-7534 (2006).

[5] Macintosh, B., Graham, J., Palmer, D., Doyon, R., Gavel, D., Larkin, J., Oppenheimer, B., Saddlemyer, L., Wallace, J. K., Bauman, B., et al., "The gemini planet imager," in [Advances in Adaptive Optics II], 6272, 62720L, International Society for Optics and Photonics (2006).

[6] Macintosh, B. A., Graham, J. R., Palmer, D. W., Doyon, R., Dunn, J., Gavel, D. T., Larkin, J., Oppenheimer, B., Saddlemyer, L., Sivaramakrishnan, A., et al., "The gemini planet imager: from science to design to construction," in [Adaptive Optics Systems], 7015, 701518, International Society for Optics and Photonics (2008).

[7] Stapelfeldt, K. R., "Extrasolar planets and star formation: science opportunities for future elts," Proceedings of the International Astronomical Union 1(S232), 149-158 (2005).

[8] N'Diaye, M., Dohlen, K., Fusco, T., and Paul, B., "Calibration of quasi-static aberrations in exoplanet direct-imaging instruments with a zernike phase-mask sensor," Astronomy \& Astrophysics 555, A94 (2013).

[9] Loupias, M., Langlois, M. P., Thiébaut, E., Delacroix, C., Leger, J., Adjali, L., and Tallon, M., "Development of an elt xao testbed using a mach-zehnder wavefront sensor: calibration of the deformable mirror," in [Unconventional Imaging and Wavefront Sensing 2015], 9617, 96170G, International Society for Optics and Photonics (2015). 
[10] Notaras, J. and Paterson, C., "Demonstration of closed-loop adaptive optics with a point-diffraction interferometer in strong scintillation with optical vortices," Optics Express 15(21), 13745-13756 (2007).

[11] Gonté, F., Araujo, C., Bourtembourg, R., Brast, R., Derie, F., Duhoux, P., Dupuy, C., Frank, C., Karban, R., Mazzoleni, R., et al., "On-sky testing of the active phasing experiment," The Messenger 136, 25-31 (2009).

[12] Surdej, I., Yaitskova, N., and Gonte, F., "On-sky performance of the zernike phase contrast sensor for the phasing of segmented telescopes," Applied optics 49(21), 4052-4062 (2010).

[13] Bharmal, N. A., Myers, R. M., Basden, A. G., and Reeves, A. P., "An interferometric wavefront sensor for high-sensitivity low-amplitude measurements," in [Adaptive Optics Systems III], 8447, 84472J, International Society for Optics and Photonics (2012).

[14] Wallace, J. K., Burruss, R. S., Bartos, R. D., Trinh, T. Q., Pueyo, L. A., Fregoso, S. F., Angione, J. R., and Shelton, J. C., "The gemini planet imager calibration wavefront sensor instrument," in [Adaptive Optics Systems II], 7736, 77365D, International Society for Optics and Photonics (2010).

[15] Love, G. D., Oag, T. J., and Kirby, A. K., "Common path interferometric wavefront sensor for extreme adaptive optics," Optics express 13(9), 3491-3499 (2005).

[16] N'Diaye, M., Vigan, A., Dohlen, K., Sauvage, J.-F., Caillat, A., Costille, A., Girard, J., Beuzit, J.-L., Fusco, T., Blanchard, P., et al., "Calibration of quasi-static aberrations in exoplanet direct-imaging instruments with a zernike phase-mask sensor-ii. concept validation with zelda on vlt/sphere," Astronomy $\mathcal{B}_{3}$ Astrophysics 592, A79 (2016).

[17] Dubost, N., Bharmal, N. A., and Myers, R. M., "Calibration of quasi-static aberrations in high-contrast astronomical adaptive optics with a pupil-modulated point-diffraction interferometer," Optics express 26(9), 11068-11083 (2018).

[18] Myers, R. M., Hubert, Z., Morris, T. J., Gendron, E., Dipper, N. A., Kellerer, A., Goodsell, S. J., Rousset, G., Younger, E., Marteaud, M., et al., "Canary: the on-sky ngs/lgs moao demonstrator for eagle," in [Adaptive Optics Systems], 7015, 70150E, International Society for Optics and Photonics (2008).

[19] Gilmore, G. F., "Opticon: a (small) part of european astronomy," in [Second Backaskog Workshop on Extremely Large Telescopes], 5382, 138-142, International Society for Optics and Photonics (2004).

[20] Loupias, M., Langlois, M., Thiébaut, E., Tallon, M., and Leger, J., "Status of an extreme adaptive optics testbench using a self-referenced mach-zehnder wavefront sensor," in [Adaptive Optics Systems V], 9909, 99094F, International Society for Optics and Photonics (2016).

[21] Bharmal, N. A., Myers, R. M., Basden, A. G., Hölck, D., and Morris, T. J., "Chough, the canary hostedupgrade for high-order adaptive optics," in [Adaptive Optics Systems IV], 9148, 91485V, International Society for Optics and Photonics (2014).

[22] Mahajan, V. N., "Strehl ratio for primary aberrations: some analytical results for circular and annular pupils," JOSA 72(9), 1258-1266 (1982).

[23] Morris, T., Gendron, E., Basden, A., Martin, O., Osborn, J., Henry, D., Hubert, Z., Sivo, G., Gratadour, D., Chemla, F., et al., "Canary phase b: on-sky open-loop tomographic lgs ao results," in [Adaptive Optics Systems IV], 9148, 91481I, International Society for Optics and Photonics (2014).

[24] Roddier, F., [Adaptive optics in astronomy], Cambridge university press (1999).

[25] Fusco, T., Ageorges, N., Rousset, G., Rabaud, D., Gendron, E., Mouillet, D., Lacombe, F., Zins, G., Charton, J., Lidman, C., et al., "NAOS performance characterization and turbulence parameters estimation using closed-loop data," in [Advancements in Adaptive Optics], 5490, 118-129, International Society for Optics and Photonics (2004). 